\title{
Contrast-enhanced multiple-phase imaging features of intrahepatic mass-forming cholangiocarcinoma and hepatocellular carcinoma with cirrhosis: A comparative study
}

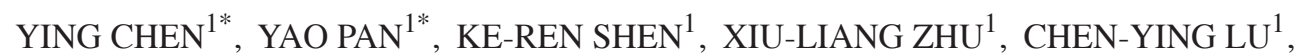 \\ QING-HAI LI ${ }^{1}$, SHU-GAO HAN ${ }^{1}$, YAN-BIAO FU ${ }^{2}$, XIU-FANG XU ${ }^{3}$ and RI-SHENG YU ${ }^{1}$ \\ ${ }^{1}$ Department of Radiology; ${ }^{2}$ Department of Pathology, The Second Affiliated Hospital, Zhejiang University School of \\ Medicine, Hangzhou 310009; ${ }^{3}$ Department of Radiology, Hangzhou Medical College, Hangzhou 310053, P.R. China
}

Received November 22, 2016; Accepted July 13, 2017

DOI: $10.3892 / 01.2017 .6656$

\begin{abstract}
The intrahepatic mass-forming cholangiocarcinoma (IMCC) is frequently misdiagnosed as hepatocellular carcinoma (HCC) in patients with cirrhosis, by numerous radiologists and clinical doctors, which results in the incorrect therapeutic treatment. A retrospective case-control study was conducted, and the contrast-enhanced multiple-phase (CEMP) computed tomography (CT) and magnetic resonance imaging (MRI) findings of 22 pathologically confirmed IMCC patients and $22 \mathrm{HCC}$ controls with underlying liver cirrhosis were analyzed at the present hospital, from January 2010 to December 2015. In addition, serum tests were conducted and clinical symptoms of patients evaluated. A statistical analysis revealed that the enhancement pattern, signal on MRI delayed phase $(\mathrm{P}<0.001)$, maximum diameter, capsule retraction, portal vein invasion, bile duct dilation and abdominal lymphadenectasis characteristics were different between IMCC and HCC patients with cirrhosis. On CEMP CT and MRI analysis, the most frequently occurring enhancement patterns of IMCC were progressive patterns $(\mathrm{P}=0.001$ or $\mathrm{P}<0.001)$. Conversely, the most frequently occurring enhancement patterns present in $\mathrm{HCC}$ were the washout patterns $(\mathrm{P}<0.001)$. Therefore, the diagnosis of IMCC in cirrhotic patients should be verified with CEMP CT and MRI analysis for the future, to determine
\end{abstract}

Correspondence to: Professor Xiu-Fang Xu, Department of Radiology, Hangzhou Medical College, 481 Binwen Road, Hangzhou 310053, P.R. China

E-mail: hzyxyxuxiufang@126.com

Professor Ri-Sheng Yu, Department of Radiology, The Second Affiliated Hospital, Zhejiang University School of Medicine, 88 Jiefang Road, Hangzhou 310009, P.R. China

E-mail: cjr.yurisheng@vip.163.com

*Contributed equally

Key words: intrahepatic mass-forming cholangiocarcinoma hepatocellular carcinoma, cirrhosis, computed tomography, magnetic resonance imaging presence or absence of progressive and/or peripheral rim-like enhancement, a hyperintensive delayed phase with capsule retraction, portal vein invasion, bile duct dilation, abdominal lymphadenectasis and increased levels of CA199.

\section{Introduction}

Intrahepatic cholangiocarcinoma (ICC) originating from biliary epithelial cells is the second most common primary intrahepatic malignancy after hepatocellular carcinoma (HCC) (1). ICC can be classified into three types on the basis of the morphology of the tumour: Mass forming, periductal infiltrating, and intraductal growing. Among which, the intrahepatic mass-forming cholangiocarcinoma (IMCC) is the most common form $(2,3)$. In recent decades, the incidence and mortality of ICC and HCC are rising markedly (4). As is known, in cirrhotic patients, the most frequent hepatic malignant tumor is HCC (5). However, evidences suggest that cirrhosis is an important risk factor for ICC $(6,7)$. It is of great importance to distinguish ICC from HCC because of the dismal prognosis of the former and different treatment options between them. For HCC, surgical resection, liver transplantation, and percutaneous ablation are all available. As for ICC, surgical resection is the only curative treatment option $(5,8)$.

Contrast-enhanced multiple-phase (CEMP) computed tomography $(\mathrm{CT})$ and magnetic resonance imaging (MRI) are widely used in investigating ICC and HCC in clinical practice. As the high incidence and various enhancement patterns of $\mathrm{HCC}$ in cirrhotic liver, any focal lesion in cirrhotic liver can be misdiagnosed as HCC by many radiologists, which may lead to inappropriate treatments (9). Therefore, it's necessary to assess and summarize the CEMP CT and MRI features of IMCC and HCC in the setting of cirrhotic liver. However, the CEMP CT and MRI findings of IMCC with hepatic cirrhosis have not been well addressed. Until now, there are only five studies (4,6,9-11) describing CT or MRI characteristics of IMCC with hepatic cirrhosis. And the comparison between IMCC and HCC in cirrhotic liver is fewer. Further, no one that discusses the imaging features of both CEMP CT and MRI findings has been published. In 
this study, we highlight the predominant CEMP CT and MRI features of IMCC and HCC in cirrhotic liver, combining the accompanying characteristics as well as serum tests to distinguish IMCC from HCC in cirrhotic liver. To the best of our knowledge, this is the first report describing both CT and MRI data for patients with IMCC in cirrhotic liver and comparison with HCC.

\section{Materials and methods}

Patients. Our institutional review board approved this retrospective study and waived the requirement for informed consent. A retrospective case-control study was conducted. The study included 22 patients ( 16 men, 6 women; mean age, 58.32 years; range, 31-69 years) with IMCC and cirrhosis consecutively registered in our hospital between January 2010 and December 2015. The enrolled criteria were as follows: i) Pathologically proven diagnosis of IMCC excluding patients with mixed hepatocellular-cholangiocarcinoma and multiple lesions; ii) availability of an abdominal CEMP CT and/or MR scans; iii) patients with cirrhosis diagnosed by imaging, pathology, or clinical criteria. CT $(n=20), \operatorname{MRI}(n=15)$ and serum tests $(n=21)$ features were retrospectively reviewed. The underlying causes of liver cirrhosis included hepatitis B $(n=13)$, alcohol abuse $(n=3)$, hepatitis $C(n=1)$, and unknown cause $(n=5)$.

The controls consisted of 22 patients (16 men, 6 women; mean age, 56.91 years; range, 34-67 years) with HCC and cirrhosis. They were matched to the IMCC cases for sex $(\mathrm{P}=1.000)$ and age $(\mathrm{P}=0.600)$. The controls were recruited during the same study period as the cases. The inclusion criteria were (1) pathology-confirmed diagnosis of HCC excluding patients with mixed hepatocellular-cholangiocarcinoma and multiple lesions; and (2), (3) the same as above. CT (n=18), MRI $(n=21)$ and serum tests $(n=22)$ features were retrospectively reviewed. The underlying causes of liver cirrhosis included hepatitis B $(n=19)$, hepatitis B with alcohol abuse $(n=2)$, and unknown cause $(n=1)$.

Image acquisition. CT imaging was performed with a multidetector-row helical CT scanners (Somatom Definition AS 40-row, Siemens Medical Systems, Erlangen, Germany) with $5 \mathrm{~mm}$ axial sections from the dome of the diaphragm to the last plane of the liver in 38 patients. All patients were examined in a fasting state with plain scanning at first, and then non-ionic contrast medium (Omnipaque $300 \mathrm{~g} / \mathrm{l}$; GE Healthcare, Milwaukee, WI, USA) $80 \mathrm{ml}$ per bolus injection was given via antecubital vein for enhanced scanning. Images were obtained separately at the arterial phase (25 sec after injection), portal venous phase (60 sec after injection) and equilibrium phase (100 sec after injection).

MR scanning was performed using a 3.0-T magnet (Signa or Discovery 750; GE Healthcare) with an eight-channel torso-array coil. Axial T1-weighted images (T1WI) and T2-weighted images (T2WI) were obtained from 36 patients, and additional contrast-enhanced T1WI (Omniscan, $0.1 \mathrm{mmol} / \mathrm{kg}$ body weight; GE Healthcare) images were obtained from all patients. Dynamic breath-hold T1WI acquisitions were obtained at arterial phase, portal venous phase, equilibrium phase and delayed phase (20-27, 45-52, 75-82 and 135-142 sec after contrast enhancement).
The imaging parameters for T1WI and T2WI were as follows: Repetition time/echo time (TR/TE) of 205/3.2 msec (or 3.9/1.8 $\mathrm{msec}$ ) and 6,000/102.5 msec (or 12,000/86.3). The matrix was $256 \times 256$, the standard field-of-view was $400 \mathrm{~mm}$ and slice thickness was $4.0 \mathrm{~mm}$ with no interslice gap. Additional diffusion weighted imaging (DWI) was performed in all patients using the following parameters: $\mathrm{TR} / \mathrm{TE}=1,300 / 60.6$ or $6,000 / 52.5 \mathrm{msec}, 5 \mathrm{~mm}$ thickness, water selective excitation for fat suppression, matrix size $=128 \times 128$, field of view $=36 \times 36 \mathrm{~cm}$, number of excitations $=6.0$, slice thickness $/$ gap $=5 \mathrm{~mm} / 1.0 \mathrm{~mm}, 20$ axial slices, scan time $=2 \mathrm{~min}$ $24 \mathrm{sec}, \mathrm{b}$ value $=0$ and $600 \mathrm{~s} / \mathrm{mm}^{2}$, under breath-hold.

Pathological examination. Histologic specimens of IMCC and HCC were obtained by percutaneous needle biopsy in 12 patients and by exploratory laparotomy and nodule biopsy in 32 patients. IMCC and HCC were diagnosed on the basis of light microscopic examinations of histologic specimens. Hematoxylin and eosin staining and immunohistochemical staining were performed on all tumors. All IMCC and HCC specimen analyses were confirmed by an experienced pathologist for diagnostic accuracy.

Image analysis. All CEMP CT and MR images were retrospectively analyzed separately by two abdominal radiologists (Y.C. and R.S.Y.) with 5 and 25 years of experience in abdominal radiology, respectively, in a blind manner. Discordance between the two was resolved by consensus. The reviewers knew that the patients had liver tumors but were unaware of pathological outcome. Each lesion was evaluated as following: The maximum diameter, morphology, location, signal and/or density of the tumors on each phase and enhancement patterns of the tumors. Accompanying findings including hepatic capsule retraction, cholangiolithiasis, bile duct dilation, portal vein invasion and lymphadenectasis were also noted. The dynamic enhancement patterns were defined as follows: i) Progressive: The nodule enhances progressively over time, reaching maximal intensity on equilibrium phase or delayed phase, including centripetal enhancement; ii) rim-like: The enhancement limited to the periphery of the lesion and remains invariable from the arterial to the portal venous and delayed phases; iii) stable: The enhancement is unmodified through the whole process but not restricted to the periphery; iv) wash-out: Intense contrast enhancement during the arterial and/or portal venous phase followed by contrast washout on equilibrium phase or delayed phase.

Statistical analysis. Statistical package for the social sciences for windows, v20.0, (SPSS, Chicago, IL, USA) was used in this statistical analysis. Categorical variables were tested using $\chi^{2}$ test or Fisher's exact test. Continous variables were compared with the independent-samples t-test if they were homogeneity of variances; otherwise, if continuous variables were heterogeneity of variances, non-parametric test was used. $\mathrm{P}<0.05$ was considered to indicate a statistically significant difference.

\section{Results}

Morphologic features of tumors. A total of 22 patients with diagnosis of IMCC with cirrhosis ranging from 2.2 to $18.1 \mathrm{~cm}$ 
Table I. Main patient and tumor features.

\begin{tabular}{lccc}
\hline Characteristic & IMCC $(\mathrm{n}=22)$ & HCC $(\mathrm{n}=22)$ & P-value \\
\hline Age & 58.32 & 56.91 & NS (0.584) \\
Sex & $16(72.7 \%)$ & $16(72.7 \%)$ & NS (1.000) \\
Man & $6(27.3 \%)$ & $6(27.3 \%)$ & 5.305 \\
Female & 8.177 & & NS (1.000) \\
Nodule size(cm) & & $7(31.8 \%)$ & $14(63.6 \%)$ \\
Location of nodules & $7(31.8 \%)$ & $1(4.6 \%)$ & \\
Left lobe & $14(63.6 \%)$ & $18(81.8 \%)$ & NS (0.176) \\
Right lobe & $1(4.6 \%)$ & $4(18.2 \%)$ & $2(9.1 \%)$ \\
Caudal lobe & $14(63.6 \%)$ & $4(18.2 \%)$ & $8(36.4 \%)$ \\
Morphology & $8(36.4 \%)$ & & 0.008 \\
Regular & $10(45.5 \%)$ & 0.002 \\
Irregular & $14(63.6 \%)$ & $<0.001$ \\
Bile duct dilation & $20(90.9 \%)$ & & \\
Portal vein invasion & & & \\
Lymphadenectasis & & & \\
\hline
\end{tabular}

\section{NS, no statistical difference.}
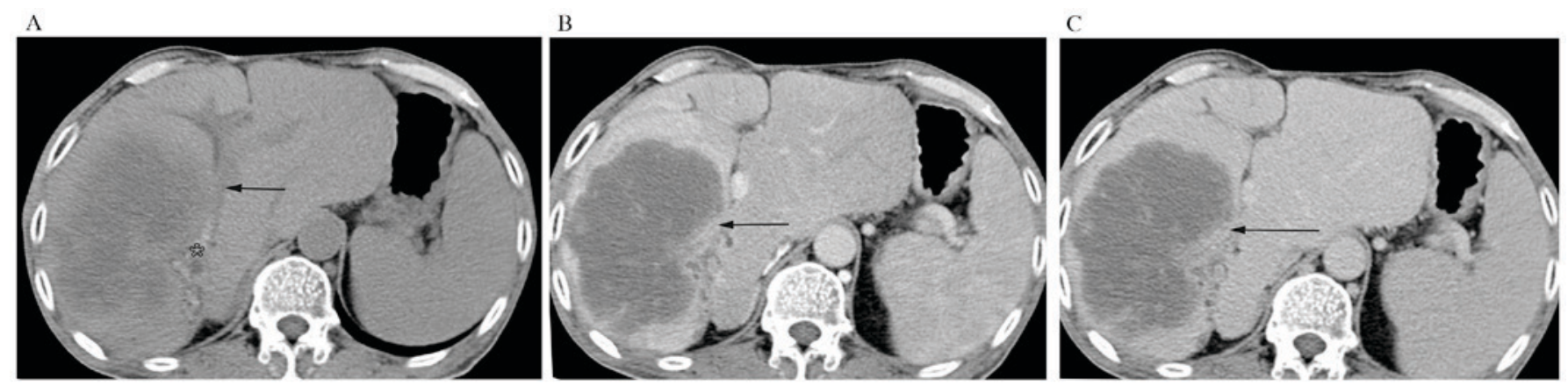

Figure 1. A 64-year-old man with IMCC and hepatic cirrhosis. (A) Unenhanced axial computed tomography scan, (B) portal phase and (C) delayed phase after intravenous contrast administration show rim-like enhancement pattern of the lesion (arrow). Accompanying characteristics include portal vein invasion and cholangiolithiasis (asterisk).

in maximum diameter (mean, $8.18 \mathrm{~cm}$ ), and 22 control patients of HCC in cirrhotic liver ranging from 1.5 to $13.1 \mathrm{~cm}$ in maximum diameter (mean, $5.31 \mathrm{~cm})$ were assessed $(\mathrm{P}=0.010)$, and the main features were summarized in Table I. The frequency of capsule retraction $(\mathrm{P}=0.001)$, portal vein invasion $(\mathrm{P}=0.002)$, bile duct dilation $(\mathrm{P}=0.007)$ and abdominal lymphadenectasis $(\mathrm{P}<0.001)$ as well as maximum diameter $(\mathrm{P}=0.010)$ were statistically significant differences.

Enhancement patterns. The enhancement patterns on CEMP CT of IMCC and HCC with cirrhosis were summarized in Table II. In the IMCC group, all the tumors appeared as hypodensity compared to the surrounding parenchyma on unenhanced scans. The analysis of the dynamic enhancement pattern throughout the different phases showed that $6(30.0 \%)$ and $11(55.0 \%)$ tumors displayed as rim-like (Fig. 1A-C) and progressive contrast enhancement. One (5.0\%) tumor had a stable enhancement pattern. The remaining $2(10.0 \%)$ tumors represented a washout pattern. In the HCC group, most
$(15,83.3 \%)$ tumors visualized on plain scans were hypodensity and the rest $(3,16.7 \%)$ tumors were isodensity compared to the surrounding parenchyma. After injection of contrast agent, $15(83.3 \%)$ tumors showed a washout enhancement pattern $(\mathrm{P}<0.001)$. One $(5.6 \%)$ and two $(11.1 \%)$ tumors have a progressive $(\mathrm{P}=0.001)$ and stable enhancement pattern, respectively. But no one having a rim-like enhancement pattern $(\mathrm{P}=0.021)$.

The enhancement patterns and hypointense signal at delayed phase on CEMP MRI of IMCC and HCC with cirrhosis were summarized in Table III. All the IMCC with cirrhosis were either hypointense $(14,93.3 \%)$ or isointense $(1,6.7 \%)$ on T1WI with a presence of homogeneous or heterogeneous hyperintense on T2WI. On DWI, all the tumors were hyperintense except one. The prevalent tumor enhancement pattern was progressive $(10,66.6 \%)$ (Fig. 2A-C). The rim-like, stable and washout (Fig. 3A-D) enhancement patterns accounted for 6.7, 6.7 and $20 \%$. As for HCC with cirrhosis, three $(14.3 \%)$ tumors appeared high-low mixed 
Table II. The enhancement patterns on CEMP CT of IMCC and HCC with cirrhosis.

\begin{tabular}{lcrr}
\hline Enhancement pattern & IMCC $(\mathrm{n}=20)(\%)$ & HCC $(\mathrm{n}=18)(\%)$ & P-value \\
\hline Rim-like & $6(30.0)$ & $0(0.0)$ & 0.021 \\
Progressive & $11(55.0)$ & $1(5.6)$ & 0.001 \\
Stable & $1(5.0)$ & $2(11.1)$ & NS $(0.595)$ \\
Wash-out & $2(10.0)$ & $15(83.3)$ & $<0.001$ \\
\hline
\end{tabular}

NS, no statistical difference.

Table III. The enhancement patterns on CEMP MRI of IMCC and HCC with cirrhosis.

\begin{tabular}{lccc}
\hline Enhancement pattern & IMCC $(\mathrm{n}=15)(\%)$ & HCC $(\mathrm{n}=21)(\%)$ & P-value \\
\hline Rim-like & $1(6.7)$ & $0(0.0)$ & NS $(0.417)$ \\
Progressive & $10(66.6)$ & $1(4.8)$ & $<0.001$ \\
Stable & $1(6.7)$ & $1(4.8)$ & NS $(1.000)$ \\
Wash-out & $3(20.0)$ & $19(90.4)$ & $<0.001$ \\
Hyperintense signal & $12(80.0)$ & & $<0.001$ \\
at delayed phase & & & \\
\hline
\end{tabular}

NS, no statistical difference.
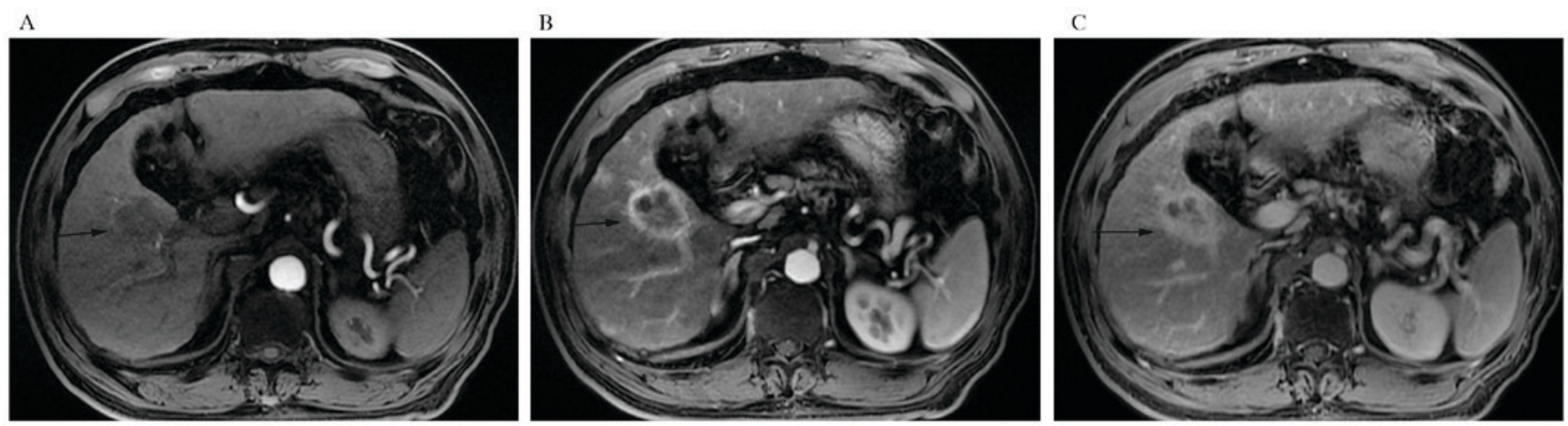

Figure 2. A 60-year-old man with IMCC and hepatic cirrhosis. Contrast-enhanced multiple-phase magnetic resonance imaging acquired on (A) arterial, (B) portal, and (C) delayed phases after intravenous contrast administration show progressive enhancement pattern of the lesion (arrow).

signals owing to hemorrhage and the remaining $(18,85.7 \%)$ were hypointense on T1WI. All the lesions were homogeneous or heterogeneous hyperintense on T2WI and hyperintense on DWI. Among these tumors, washout (Fig. 4A and B) was the most common type throughout the different phases of the study $(19,90.4 \%)(\mathrm{P}<0.001)$. And there were respectively $1(4.8 \%)$ and $1(4.8 \%)$ displaying progressive $(\mathrm{P}<0.001)$ and stable enhancement patterns with no one having a rim-like enhancement pattern.

In addition, we found $12(80.0 \%)$ of the 15 IMCC lesions while only 2 (9.5\%) of $21 \mathrm{HCC}$ lesions with cirrhosis appearing as hyperintense at delayed phases on CEMP MRI $(\mathrm{P}<0.001)$.

Tumor markers detection. There were 21 patients with IMCC and 22 patients with HCC having a record of tumor markers detection (Table IV). The average \pm SD numbers of CA199, AFP and CEA were 1497.53 $\pm 3626.50 \mathrm{U} / \mathrm{ml}, 517.92 \pm 2047.69$ and 58.04 \pm 225.09 respectively in IMCC group. While, the average \pm SD numbers of CA199, AFP and CEA were $25.26 \pm 30.29 \mathrm{U} / \mathrm{ml}, 5460.10 \pm 17028.11$ and $2.94 \pm 1.65 \mathrm{ng} / \mathrm{ml}$, respectively in $\mathrm{HCC}$ group. These continuous variables were heterogeneity of variances, so non-parametric test was used. There were statistically differences in CA199 $(\mathrm{P}=0.022)$ and AFP $(\mathrm{P}=0.013)$ between IMCC group and HCC group, but no statistically difference in CEA between the two groups.

\section{Discussion}

Cholangiocarcinoma arises from the ductular epithelium of the biliary tree first reported by Durand Fardel in 1840. It is classified into intrahepatic (including hilar) and extrahepatic cholangiocarcinoma (12). ICC is the second most common primary hepatic tumor after HCC, which accounts for $15-20 \%$ of all primary hepatic tumors (13). And IMCC is the most common 
Table IV. Tumor markers detection of IMCC and HCC with cirrhosis.

\begin{tabular}{lccr}
\hline Tumor marker & IMCC $(\mathrm{n}=21)$ & HCC $(\mathrm{n}=22)$ & P-value \\
\hline CA199 $(\mathrm{U} / \mathrm{ml})$ & $1,497.53 \pm 3626.50$ & $25.26 \pm 30.29$ & 0.022 \\
AFP $(\mathrm{ng} / \mathrm{ml})$ & $517.92 \pm 2047.69$ & $5,460.10 \pm 17,028.11$ & 0.013 \\
CEA $(\mathrm{ng} / \mathrm{ml})$ & $58.04 \pm 225.09$ & $2.94 \pm 1.65$ & 0.076
\end{tabular}

CA199, carbohydrate antigen 199; AFP, alphafetoprotein; CEA, carcinoembryonic antigen.
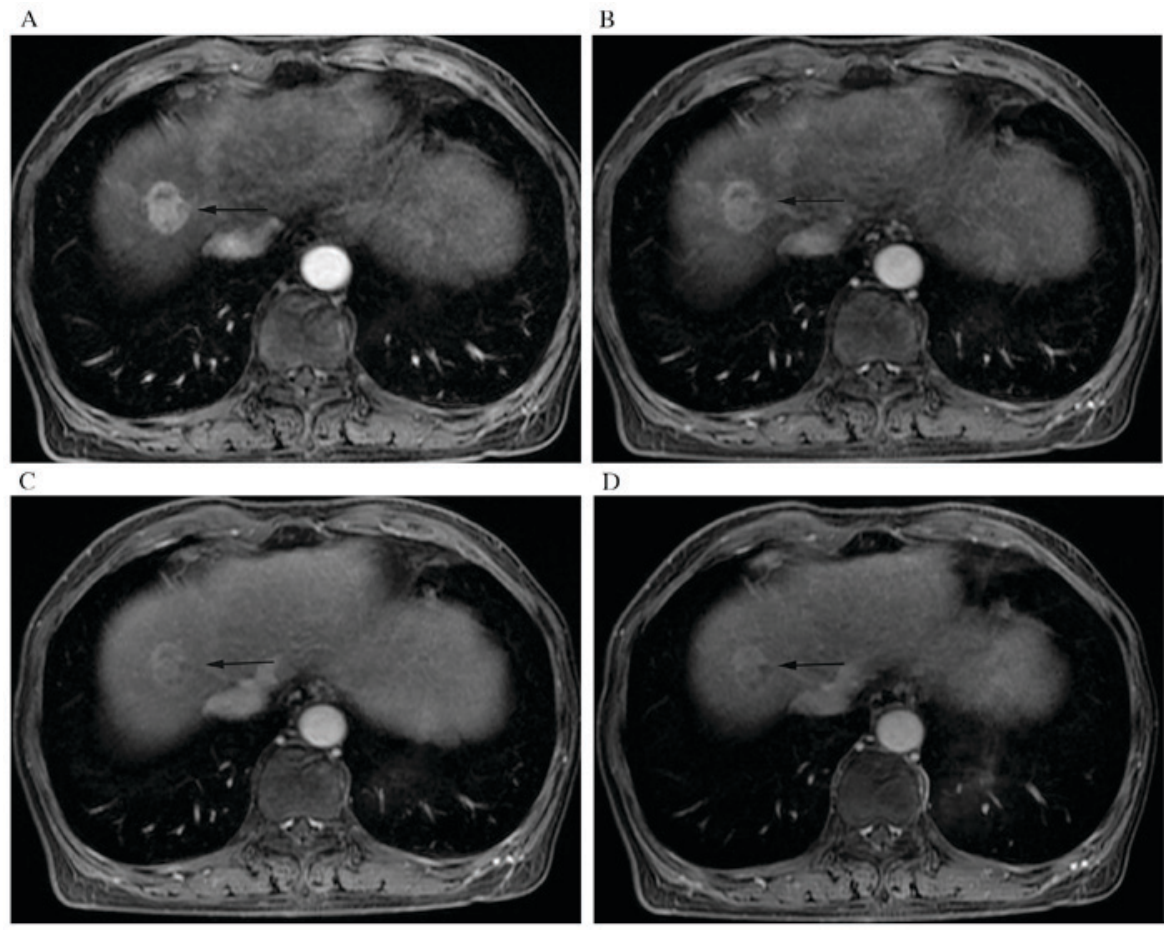

Figure 3. A 63-year-old woman with IMCC and hepatic cirrhosis. Contrast-enhanced multiple-phase magnetic resonance imaging of (A) arterial, (B) portal, (C) equilibrium and (D) delayed phases after intravenous contrast administration show wash-out enhancement pattern of the nodule (arrow). On delayed phase the nodule remains hyperintense compared with surrounding liver parenchyma.
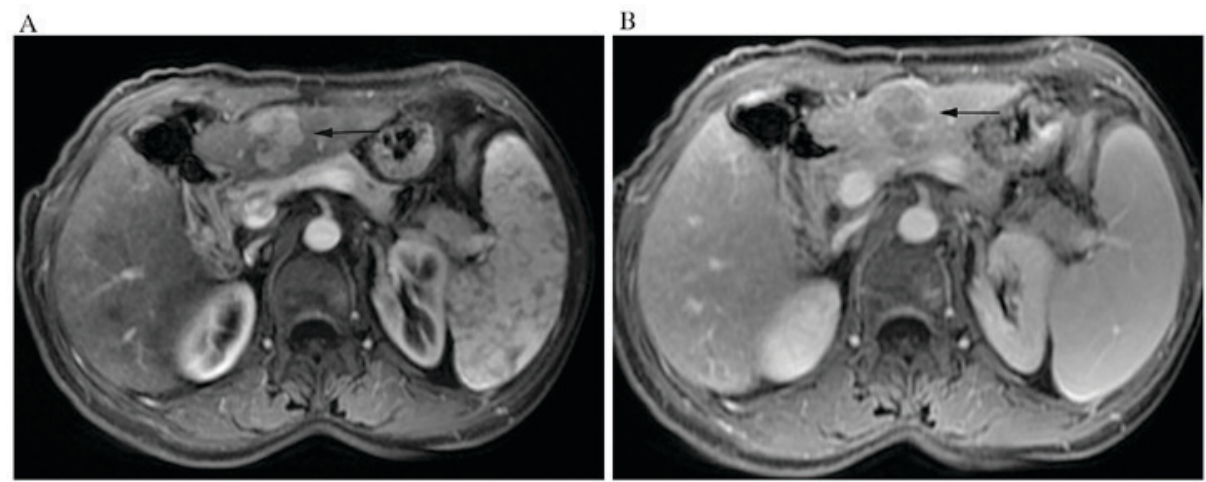

Figure 4. A 57-year-old woman with HCC and hepatic cirrhosis. The nodule (arrow) displays wash-out enhancement pattern. On (A) arterial phase, the nodule appears significantly hyperintense, while on (B) delayed phases, it was hypointense compared with surrounding liver parenchyma.

type of ICC and clinically is comparable to HCC (14). With respect to HCC, IMCC has dismal prognosis and limited choices of treatment. Therefore, it's critical to accurately distinguish the two entities (6). However, the discrimination between IMCC and $\mathrm{HCC}$ in cirrhotic patients remains challenging because of atypical enhancement patterns of IMCC and HCC (15). Our research may provide some valuable informations for distinguishing IMCC from HCC in patients with cirrhosis. 
In the present study, the mean diameter of IMCCs was larger than that of HCCs, and there was statistically significant difference $(\mathrm{P}=0.010)$, which was not comparable with other reports in the literature (9). We considered that the reason might be that IMCC patients were usually silent in clinical and patients were discovered with symptoms frequently at an advanced stage (8). While HCC patients were usually discovered by physical examination. As we could see in our cases, there were 13 patients with IMCC having symptoms of abdominal pain, abdominal discomfort or feeble. However, only 4 HCC patients were symptomatic and the remaining were discovered by physical examination.

The CEMP CT and MRI results of our research showed that the most prevalent enhancement pattern of IMCC in the setting of cirrhosis was progressive enhancement (CT 55.0\% and MRI $66.6 \%$ ), while only $5.6 \%$ (CT) and $4.8 \%$ (MRI) in $\mathrm{HCC}$ group, and there were statistically significant differences $(\mathrm{P}=0.001$ and $\mathrm{P}<0.001)$, which were not only consistent with other observations in the setting of cirrhosis $(4,6,9,11)$ but also resembled as previously reported in normal livers $(3,13,16)$. According to this enhancement pattern, radiologists could differentiate IMCC from HCC in patients with cirrhosis, which appeared peripheral hyperdensity/hyperintensity at hepatic arterial phase (HAP) or partial hyperdensity/hyperintensity with gradual and centripetal enhancement during later phases. According to our observation and a previous report (17) that the HAP peripheral or partial hyperintensity was owing to the greater density of viable tumor cells, and the centripetal progressive enhancement was attributed to the variable degree of fibrosis in the center of IMCC.

The second prevalent enhancement pattern of IMCC in the setting of cirrhosis on CEMP CT was rim-like enhancement (30.0\%), while no one in HCC group. Noticeably, there was statistically significant difference $(\mathrm{P}=0.021)$, which was also demonstrated in the research of Kim et al (9) and Iavarone et al (11), indicating that this pattern was related to tumor size, mostly seen in large lesions $\geq 3 \mathrm{~cm}$, which supported our results, and this provided significant information for diagnosis IMCC in patients with cirrhosis. However, interestingly, we noticed that two IMCC lesions showing rim-like enhancement on CEMP CT were progressive enhancement on CEMP MRI. Previous studies $(11,18)$ also demonstrated the discrepancies in the diagnostic accuracy between CT-scan and MRI, considering it was owing to the stronger arterial uptake and greater sensitivity for intravascular contrast agent on MRI than CT. So we thought that equivocal imaging patterns need to be confirmed by MRI for further examination.

Although some previous researches $(6,11)$ showing that there was no IMCC having a hallmark of HCC which represented by hyperenhancement on the arterial phase followed by contrast wash-out on the portal venous and/or delayed phase, we observed that 10.0 and $20.0 \%$ IMCCs displayed wash-out enhancement pattern on CEMP CT and MRI in our study $(\mathrm{P}<0.001)$, which may lead to misdiagnosis with HCCs, which was also found in some recent reports $(4,9)$. What's more, we also noticed that the signals at delayed phase of the IMCCs with wash-out enhancement pattern were hyperintense, while, the overwhelming majority HCCs were observed hypointense at delayed phase. And there was statistically significant difference in hyperintense at delayed phases on CEMP MRI $(\mathrm{P}<0.001)$, which was of great help in making correct diagnoses of the two entities in patients with cirrhosis. To the best of our knowledge, there have not been described previously in the English language literature.

In addition, there were 5.0 and $6.7 \%$ IMCCs in our research manifested stable enhancement pattern on CEMP CT and MRI, which were also found in other observations (4) on CEMP MRI and indicated that this stable enhancement pattern was related to tumor size and was mostly seen in small lesions $<3 \mathrm{~cm}$. And there was no significant difference between IMCC and HCC which might result in increasing risk of misdiagnosis, if radiologists didn't take notice to this potential similarity between IMCC and HCC.

Accompanying characteristics also played an important role in diagnosis of IMCC in cirrhotic liver. IMCC usually have an appearance of capsule retraction. Some researchers thought scirrhous stroma of ICC was related to the imaging of capsular retraction (19). Resembling the classic findings of IMCC in noncirrhotic liver, bile duct dilatation is also a characteristic of IMCC in cirrhotic liver, because IMCC arises from the ductular epithelium of the biliary tree and regularly accompanies with bile duct wall infiltration leading to bile ducts stricture or blocking while the adjacent bile ducts dilatate. Another reason is IMCC occasionally coexisted with hepatolithiasis which also results in bile duct dilatation. Narrowing or obstruction of portal vein is frequently seen in IMCC due to invasion or external compression. In the present study, we found that $63.6 \%$ IMCCs had a sign of portal vein invasion and only $9.1 \% \mathrm{HCCs}$ $\operatorname{did}(\mathrm{P}=0.008)$. The accompanying characteristics above were comparable with other reports in the literature $(4,6,9,11)$ and could help to suggest the correct diagnoses of the two tumors. Another difference between IMCCs and HCCs in cirrhotic liver was lymphadenectasis. Images showed 90.9\% IMCCs in our research accompanied with lymphadenectasis in porta hepatis, retroperitoneum or cardiodiaphragmatic angle, while only $36.4 \%$ HCCs had lymphadenectasis and no one showed lymphadenectasis in cardiodiaphragmatic angle, which were not been described previously in the English language literature, to the best of our knowledge.

As is known, AFP is a specific tumor marker of HCC. However, there are no tumour markers specific for cholangiocarcinoma. But some can be used as a diagnostic guide such as CA199 and CEA (12). Previous papers reported that the sensitivity and specificity of CA199 concentrations of more than $100 \mathrm{U} / \mathrm{ml}$ in diagnosing cholangiocarcinoma were relatively high $(12,20)$. In our cases, compared with HCCs group, expression level of CA199 significantly increased in IMCCs group $(\mathrm{P}=0.022)$, which might be useful in correct diagnosis of IMCC, but was not in conformity with the research of Sheng et al (4), and further studies were needed.

We acknowledge that our study had several limitations. Firstly, the sample size of IMCCs was relatively small but previous study also included small samples, which reflected the low incidence (less that 5\%) of ICC in patients with cirrhosis. Secondly, it was a retrospective study and selection bias might exist. Thirdly, there was no data about diagnostic sensitivity and specificity of CEMP CT and MRI in differential diagnosis owing to the retrospective nature and further prospective studies would be needed. 
In conclusion, CEMP MRI is more advantageous over CT in displaying the imaging features of progressive enhancement. The CEMP CT and MRI features may be able to aid in differentiating IMCC and HCC in cirrhosis and choice of appropriate treatment strategy. It should be seriously considered the diagnosis of IMCC in cirrhotic patients that progressive and/or peripheral rim-like enhancement on CEMP CT and MRI, hyperintense at delayed phase on CEMP MRI with capsule retraction, portal vein invasion, bile duct dilation, abdominal lymphadenectasis and CA199 elevated.

\section{Acknowledgements}

This study was supported by funding from the Medical Science and Technology Project of the Health Department of Zhejiang Province of China (no. 2016147345).

\section{References}

1. Tyson GL and El-Serag HB: Risk factors for cholangiocarcinoma. Hepatology 54: 173-184, 2011.

2. Kim SA, Lee JM, Lee KB, Kim SH, Yoon SH, Han JK and Choi BI: Intrahepatic mass-forming cholangiocarcinomas: Enhancement patterns at multiphasic CT, with special emphasis on arterial enhancement pattern-Correlation with clinicopathologic findings. Radiology 260: 148-157, 2011.

3. Lim JH: Cholangiocarcinoma: Morphologic classification according to growth pattern and imaging findings. AJR Am J Roentgenol 181: 819-827, 2003.

4. Sheng RF, Zeng MS, Rao SX, Ji Y and Chen LL: MRI of small intrahepatic mass-forming cholangiocarcinoma and atypical small hepatocellular carcinoma $(\leq 3 \mathrm{~cm})$ with cirrhosis and chronic viral hepatitis: A comparative study. Clin Imaging 38 265-272, 2014.

5. Forner A, Llovet JM and Bruix J: Hepatocellular carcinoma. Lancet 379: 1245-1255, 2012.

6. Rimola J, Forner A, Reig M, Vilana R, de Lope CR, Ayuso C and Bruix J: Cholangiocarcinoma in cirrhosis: Absence of contrast washout in delayed phases by magnetic resonance imaging avoids misdiagnosis of hepatocellular carcinoma. Hepatology 50: 791-798, 2009.

7. Palmer WC and Patel T: Are common factors involved in the pathogenesis of primary liver cancers? A meta-analysis of risk factors for intrahepatic cholangiocarcinoma. J Hepatol 57: 69-76, 2012.

8. Blechacz B and Gores GJ: Cholangiocarcinoma: Advances in pathogenesis, diagnosis, and treatment. Hepatology 48: 308-321, 2008 .
9. Kim SJ, Lee JM, Han JK, Kim KH, Lee JY and Choi BI: Peripheral mass-forming cholangiocarcinoma in cirrhotic liver. AJR Am J Roentgenol 189: 1428-1434, 2007.

10. Quaia E, Angileri R, Arban F, Gennari AG and Cova MA: Predictors of intrahepatic cholangiocarcinoma in cirrhotic patients scanned by gadobenate dimeglumine-enhanced magnetic resonance imaging: Diagnostic accuracy and confidence. Clin Imaging 39: 1032-1038, 2015.

11. Iavarone M, Piscaglia F, Vavassori S, Galassi M, Sangiovanni A, Venerandi L, Forzenigo LV, Golfieri R, Bolondi L and Colombo M: Contrast enhanced CT-scan to diagnose intrahepatic cholangiocarcinoma in patients with cirrhosis. J Hepatol 58: 1188-1193, 2013.

12. Khan SA, Thomas HC, Davidson BR and Taylor-Robinson SD: Cholangiocarcinoma. Lancet 366: 1303-1314, 2005.

13. Ciresa M, De Gaetano AM, Pompili M, Saviano A, Infante A, Montagna M, Guerra A, Giuga M, Vellone M, Ardito F, et al: Enhancement patterns of intrahepatic mass-forming cholangiocarcinoma at multiphasic computed tomography and magnetic resonance imaging and correlation with clinicopathologic features. Eur Rev Med Pharmacol Sci 19: 2786-2797, 2015.

14. Kang Y, Lee JM, Kim SH, Han JK and Choi BI: Intrahepatic mass-forming cholangiocarcinoma: Enhancement patterns on gadoxetic acid-enhanced MR Images. Radiology 264: 751-760, 2012.

15. Kim R, Lee JM, Shin CI, Lee ES, Yoon JH, Joo I, Kim SH, Hwang I, Han JK and Choi BI: Differentiation of intrahepatic mass-forming cholangiocarcinoma from hepatocellular carcinoma on gadoxetic acid-enhanced liver MR imaging. Eur Radiol 26: 1808-1817, 2016.

16. Maetani Y, Itoh K, Watanabe C, Shibata T, Ametani F, Yamabe H and Konishi J: MR imaging of intrahepatic cholangiocarcinoma with pathologic correlation. AJR Am J Roentgenol 176: 1499-1507, 2001

17. Chung YE, Kim MJ, Park YN, Choi JY, Pyo JY, Kim YC, Cho HJ Kim KA and Choi SY: Varying appearances of cholangiocarcinoma: Radiologic-pathologic correlation. Radiographics 29: 683-700, 2009.

18. Semelka RC, Martin DR, Balci C and Lance T: Focal liver lesions: Comparison of dual-phase $\mathrm{CT}$ and multisequence multiplanar MR imagingincluding dynamic gadolinium enhancement. J Magn Reson Imaging 13: 397-401, 2001.

19. Tsunematsu S, Chuma M, Kamiyama T, Miyamoto $N$, Yabusaki S, Hatanaka K, Mitsuhashi T, Kamachi H, Yokoo H, Kakisaka T, et al: Intratumoral artery on contrast-enhanced computed tomography imaging: Differentiating intrahepatic cholangiocarcinoma from poorly differentiated hepatocellular carcinoma. Abdom Imaging 40: 1492-1499, 2015

20. Mosconi S, Beretta GD, Labianca R, Zampino MG, Gatta G and Heinemann V: Cholangiocarcinoma. Crit Rev Oncol Hematol 69: 259-270, 2009. 